\title{
Performance Limitations of 40-Gb/s RZ-DPSK DWDM Systems Due to Nonlinear Effects and Their Mitigation
}

\author{
M. Arafat Rahman Khan, Tahsin Faruque, and Mohammad Faisal
}

\begin{abstract}
Performance of $40-\mathrm{Gb} / \mathrm{s}$ return-to-zero differential phase-shift keying (RZ-DPSK) dense wavelength division multiplexed (DWDM) systems is evaluated numerically. The quality factor $Q$ is measured at the receiving end of the dispersion-managed (DM) transmission line. The performance margin due to fiber nonlinear effects like crossphase modulation and four-wave mixing has been investigated. The mitigation technique for these nonlinear effects has also been explored with satisfactory results.
\end{abstract}

Index Terms-Cross-phase modulation (XPM), dispersion management, DWDM, four-wave mixing (FWM), RZ-DPSK.

\section{INTRODUCTION}

Advanced modulation formats particularly phase modulation formats like differential phase-shift keying (DPSK), differential quadrature phase-shift keying (DQPSK) schemes have attracted huge research attention because of their many advantages over conventional on-off keying (OOK) scheme in fiber-optic communication systems [1][4]. Traffic on Internet has increased very rapidly and various telecommunication and multimedia applications are continuously increasing the data rate. Fiber-optic communication will handle this huge ever-increasing capacity and PSK modulation techniques will be employed for ultra-high speed long-haul lightwave transmission. RZDPSK and higher multilevel differential PSK formats are being studied extensively as they are highly prospective [5][9]. Though RZ-DQPSK is promising as it has higher spectral efficiency, it is complex and costly. On the other hand, RZ-DPSK has better prospect for next generation fiber-optic communication system due to its simplicity and low cost. Our recent work shows that DPSK is more attractive than DQPSK and 8-DPSK in many cases like higher tolerance to chromatic dispersion (up to a certain limit) and nonlinearity and it can handle higher bit rate and transmission reach [5]. Furthermore, RZ-DPSK has many advantages over NRZ with higher noise tolerance and resistance to nonlinear effects [10]. Carrier-suppressedreturn-to-zero DPSK (CSRZ-DPSK) is another propitious candidate for high-speed long-haul fiber-optic communication. CSRZ-DPSK offers more tolerance to filtering and chromatic dispersion because of its narrow spectrum [11], [12], however, recent works show that RZDPSK format is more advantageous in many other respects [13]-[14]. Even RZ-DPSK shows a convincing comparable

Manuscript received January 25, 2014; revised April 22, 2014.

The authors are with Bangladesh University of Engineering and Technology, Dhaka - 1000, Bangladesh (e-mail: arafat2138@gmail.com, tahsin_aninda@yahoo.com,mdfaisal@eee.buet.ac.bd). performance in case of chromatic dispersion tolerance with increased free-spectral-range (FSR) of Delay Interferometer (DI) and with narrow filtering [15].

RZ-DPSK is the most suitable choice for high-speed long-haul lightwave transmission among the advanced modulation formats. Both linear and nonlinear performance analyses of RZ-DPSK in WDM and DWDM systems are necessary. Performance of RZ-DPSK was evaluated and compared with that of RZ-OOK in 10-Gb/s DWDM dispersion-managed (DM) transmission system [3]. Performance of OOK, DPSK and DQPSK were experimentally measured and compared with and without RZ carving for a DWDM system, where RZ-DPSK was found to be the optimal choice among the formats for 40$\mathrm{Gb} / \mathrm{s}$ bit rate per channel [16]. However, the authors considered only two channels $50 \mathrm{GHz}$ apart and they emphasized on filtering. Nonlinear performance of ASK and DPSK has been compared for single-channel and 4-ch DWDM system using different types of fiber [17]. Spectral efficiency and nonlinear tolerance are also investigated for DPSK formats in 5-ch 160-Gb/s WDM systems using Raman amplifiers [11]. Spectral performance of RZ-OOK and RZ-DPSK has been compared in OTDM-WDM systems and found that RZ-DPSK outperformed RZ-OOK up to $4 \mathrm{~dB}$ for all bit rates [2]. Most of these works consider only few channels which are not sufficient for evaluating nonlinear effects properly. Nonlinear effects, mainly, cross-phase modulation (XPM) and four-wave mixing (FWM) increase drastically with the increase in number of channels. To the best of our knowledge, few studies have been reported on nonlinear performance of $40 \mathrm{~Gb} / \mathrm{s}$ RZ-DPSK DWDM systems including periodic dispersion compensation and amplification using erbium-doped fiber amplifiers (EDFA).

In this paper, we numerically investigate the performance degradation of RZ-DPSK DWDM systems due to nonlinear effects like XPM and FWM. The duty cycle is assumed as $66 \%$ and per channel bit rate is $40-\mathrm{Gb} / \mathrm{s}$. The channel count is varied up to $33-\mathrm{ch}$. The $Q$-factor is measured at the receiver which is placed at the end of DM transmission line and the launch power, transmission reach, channel count and channel spacing are varied. OPTISYSTEM simulation tool is used to model the system and estimate the $Q$. At the end we also investigate the reduction of these nonlinear effects for different number of channels.

\section{THEORETICAL MODELLING}

The optical signal propagating in fiber is governed by the nonlinear Schrödinger equation (NLSE) which is solved numerically using OPTISYSTEM 12 simulation tool. The NLSE for a propagating channel can be written as 


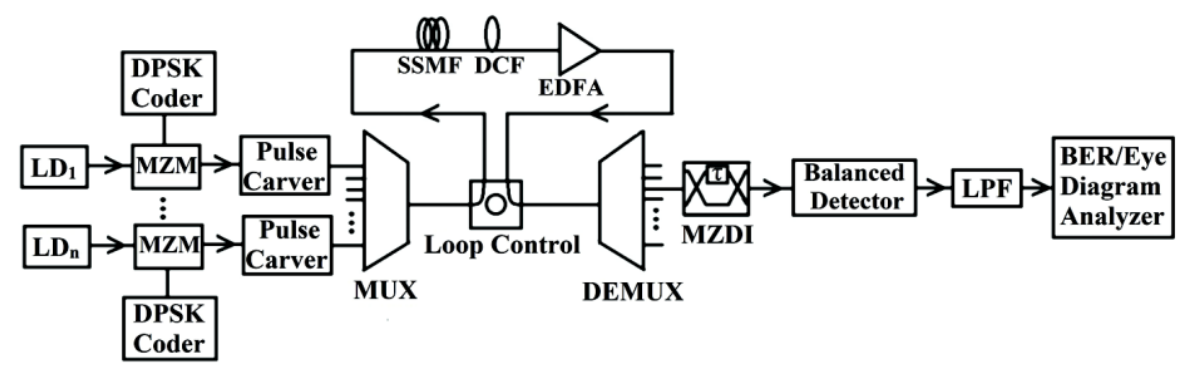

Fig. 1. System model.

$$
i \frac{\partial \mathrm{U}}{\partial \mathrm{Z}}+\frac{D(Z)}{2} \frac{\partial^{2} U}{\partial T^{2}}+\gamma(Z)|U|^{2} U=-i \alpha(Z) U+i g(Z) U
$$

where $U$ is the slowly varying envelope of the optical pulse, $Z$ is transmission distance; $D(Z), \gamma(Z), \alpha(Z)$ and $g(Z)$ represent fiber chromatic dispersion, nonlinearity, fiber loss and amplifier gain, respectively. The total system model is shown in Fig. 1 which works on the basis of (1). The transmitter section consists of laser sources, DPSK encoders following Mach-Zehnder Modulators (MZM), pulse carvers and multiplexer. The channel wavelengths are allocated with equal channel spacing and the center channel is placed at $1552.524 \mathrm{~nm}$ or $(193.1 \mathrm{THz})$ following $100 \mathrm{GHz}$ ITU grid. Each channel carries a pseudorandom bit sequence (PRBS) with length of $2^{7}-1$. The differentially encoded bit sequence is used to modulate the phase of carrier from laser source using MZM and then pulse carver is used to achieve RZ signal with desired duty cycle. All modulated channels are multiplexed and transmitted through the periodically amplified DM transmission line. Each span comprises of standard single-mode fiber (SSMF) following dispersion compensating fiber (DCF) and an EDFA at end of span. The DM map is same as span length, which is $60 \mathrm{~km}$ and there is no residual dispersion at the end of each span. The loop (DM map) is repeated as many times as necessary to cover the desired transmission reach. The fiber and system parameters are given in Table I. The amplifier gain is adjusted to compensate for the loss for each span. Noise figure of EDFA is $6 \mathrm{~dB}$. The polarization state of all channels is assumed identical. Polarization mode dispersion (PMD) and polarization dependent loss (PDL) are ignored.

\begin{tabular}{ccc} 
TABLE I: FIBER AND SYSTEM PARAMETERS \\
\hline \hline Parameter & SSMF & DCF \\
\hline Attenuation, $\alpha[\mathrm{dB} / \mathrm{km}]$ & 0.2 & 0.5 \\
Dispersion, D [ps/nm/km] & 16.75 & -83.25 \\
Fiber length, $[\mathrm{km}]$ & 50 & 10 \\
Nonlinearity coefficient, $\gamma[1 / \mathrm{W} / \mathrm{km}]$ & 1.32 & 4.78 \\
Effective area of core, $\mathrm{A}_{\text {eff }}\left[\mu \mathrm{m}^{2}\right]$ & 80 & 22 \\
PMD Coefficient, $[\mathrm{ps} / \sqrt{\mathrm{km}}]$ & 0.5 & 0.5 \\
Center wavelength, $\lambda[\mathrm{nm}]$ & \multicolumn{2}{c}{1552.524} \\
Data rate per ch, $[\mathrm{Gb} / \mathrm{s}]$ & \multicolumn{2}{c}{00} \\
Spectral Efficiency, $[\mathrm{b} / \mathrm{s} / \mathrm{Hz}]$ & \multicolumn{2}{c}{0.66} \\
\hline \hline
\end{tabular}

The demultiplexed channels are demodulated and reconstructed at the receiver. A one-bit delay Mach-Zehnder Delay Interferometer (MZDI) and a balanced detector are employed to demodulate the RZ-DPSK signals. A secondorder electrical Bessel low pass filter (LPF) is used at the end. The MUX/DEMUX filter characteristics are much important to design WDM/DWDM systems [18]. The $3 \mathrm{~dB}$ bandwidth of MUX/DEMUX filter is optimized in order to obtain highest receiver sensitivity and spectral efficiency of $0.66 \mathrm{~b} / \mathrm{s} / \mathrm{Hz}$ has been achieved.

Eye-pattern is achieved numerically at the receiver-end and $Q$-factor of the received signal is measured. The $Q$ factor represents the statistical variation in the received signal and is defined by,

$$
Q=\frac{\left|m_{0}-m_{1}\right|}{\sigma_{0}+\sigma_{1}}
$$

where, $m_{0}$ and $m_{1}$ are the mean and $\sigma_{o}$ and $\sigma_{1}$ are the standard deviation of the received signal measured for binary data " 0 " and " 1 ", respectively.

Eye Opening Penalty (EOP) is defined as the ratio of eye height of the single channel to that of multiple channels and is measured in decibel. It can be expressed as

$$
E O P=10 \log \frac{E O_{s c}}{E O_{m c}}
$$

where, $E O_{s c}$ and $E O_{m c}$ are the eye-opening height of the single channel and multiple channels, respectively. Different system parameters like launch power, distance, channel count and channel spacing are varied to observe the impact of nonlinear effects on system performance.

\section{PERFORMANCE ANALYSES OF DWDM SySTEMS}

The system performance of 66\% RZ-DPSK DWDM systems is investigated at $40-\mathrm{Gb} / \mathrm{s}$ per channel and typical channel spacing is taken as $100 \mathrm{GHz}$. In all cases, the worst channel is observed for measuring $Q$-factor using (2).

In the first simulation $Q$-factor is evaluated by increasing the number of channels from 1 to 33 . The total number of span is 6 , so the total transmission reach is $360 \mathrm{~km}$ and input power is $3 \mathrm{~mW}$. It is quite evident from Fig. 2 that if we increase the number of channels, the $Q$-factor decreases. In case of single channel, there is less nonlinear effect, only self-phase modulation (SPM) hence the $Q$-factor is much better. As we increase the number of channels, optical signal to noise ratio (OSNR) decreases due to adverse nonlinear effect i.e. addition of XPM and FWM. From the figure we see that $Q$-factor is equal to and above $6 \mathrm{~dB}\left(\mathrm{BER}=10^{-9}\right.$ for $Q=6$ ) up to 22 channels. After that $Q$ goes below that margin. Nonlinear effects, mainly XPM and FWM increase with increase of number of channels, however, SPM effect might remain same as it affect single channel only. Fig. 3 
shows four eye-diagrams for different number of channels. System performance can also be directly estimated from eye-diagrams and eye-opening penalty (EOP) can be calculated using (3). Taking singe-channel eye-diagram as reference, EOP is calculated as $2.04 \mathrm{~dB}, 3.34 \mathrm{~dB}$ and 6.02 $\mathrm{dB}$ for 9-ch, 19-ch and 33-ch DWDM systems, respectively. It is obvious from the diagrams that eye-opening reduces (EOP increases) with the increase of number of channels, i.e. performance degrades gradually which is also noticed in Fig. 2 and the reason is mainly nonlinear impairments.

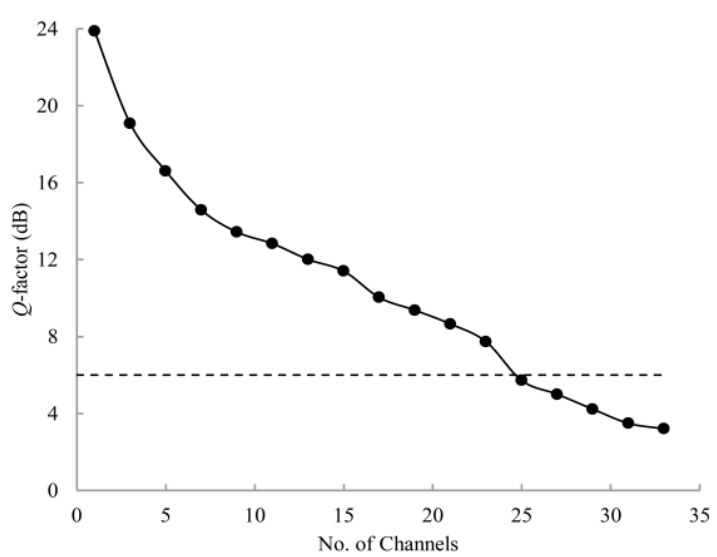

Fig. 2. $Q$-factor versus number of channels for $100 \mathrm{GHz}$ DWDM system.

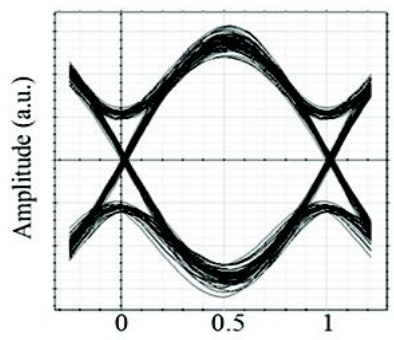

(a)

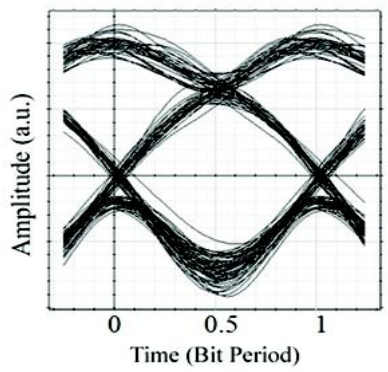

(c)

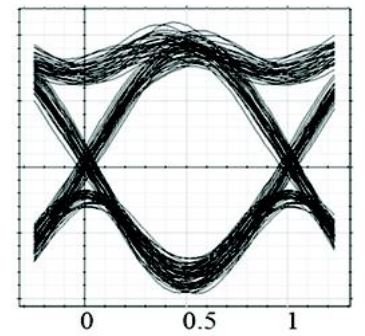

(b)

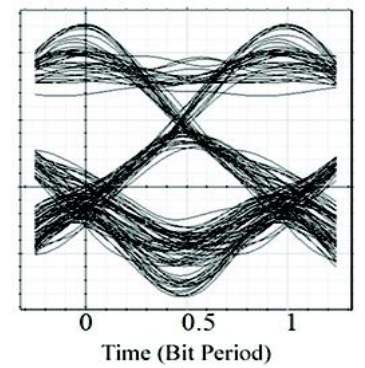

(d)
Fig. 3. Eye-diagrams for various channels ( 1 Bit Period $=25$ ps): (a) Single channel, (b) 9-channel, (c) 19-channel and (d) 33-channel.

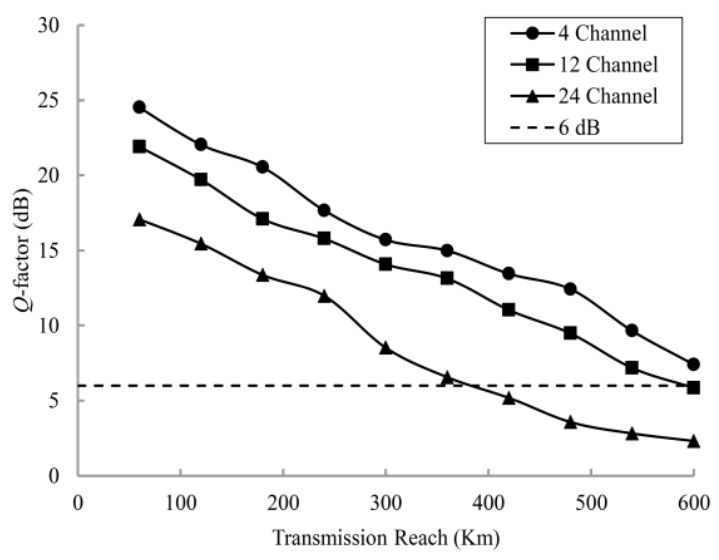

Fig. 4. $Q$-factor versus transmissions reach.
Fig. 4 shows $Q$-factor as a function of transmission reach. For DWDM system, $Q$ decreases to $6 \mathrm{~dB}$ at around $380 \mathrm{~km}$. Here 4-ch DWDM is less affected by nonlinear effects as number of channel is very low, its $Q$-value is well above 6 $\mathrm{dB}$ up to $600 \mathrm{~km}$. On the other hand, 24-ch DWDM is mostly impaired by XPM and FWM.

To observe the impact of fiber nonlinear effects more profoundly, we conduct the simulation for 21-ch DWDM system with back-to-back configuration and with $360 \mathrm{~km}$ transmission reach. Obviously for 21-ch, $Q$ will decrease rapidly with distance. Now $Q$ is plotted against launch power per channel which is varied from $1 \mathrm{~mW}$ to $12 \mathrm{~mW}$ and the result is shown in Fig. 5. For back-to-back system, $Q$-values remain almost same, whereas $Q$ reduces from 10.33 $\mathrm{dB}$ to $5.22 \mathrm{~dB}$ after transmission of $360 \mathrm{~km}$. For lower values of input power, system performs better and $Q$-values remain much above $6 \mathrm{~dB}$, but after $10 \mathrm{~mW}, Q$ goes below that margin. The performance degradation is mainly due to XPM and FWM effects, and it is noted that chromatic dispersion has a little influence on system performance as it is perfectly compensated at the end of each span. Fiber nonlinear effects are highly dependent on launch power and transmission distance [19].

So far channel spacing is kept $100 \mathrm{GHz}$, now we vary the channel spacing from $50 \mathrm{GHz}$ to $200 \mathrm{GHz}$ and evaluate the performance. The channel spacing is maintained larger than modulated signal bandwidth. In case of lower channel spacing like $50 \mathrm{GHz}$, MUX/DEMUX filter bandwidth is required to be readjusted to attain better receiver sensitivity. For $50 \mathrm{GHz}$ spacing, spectral efficiency is enhanced to 0.8 b/s/Hz. The result is plotted in Fig. 6 .

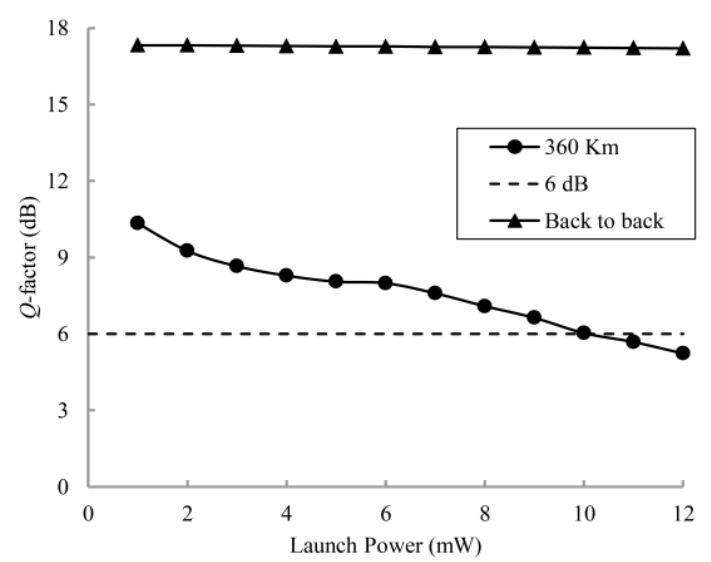

Fig. 5. $Q$-factor versus launch power for a $360 \mathrm{~km}$ long DWDM and with a back-to-back configuration.

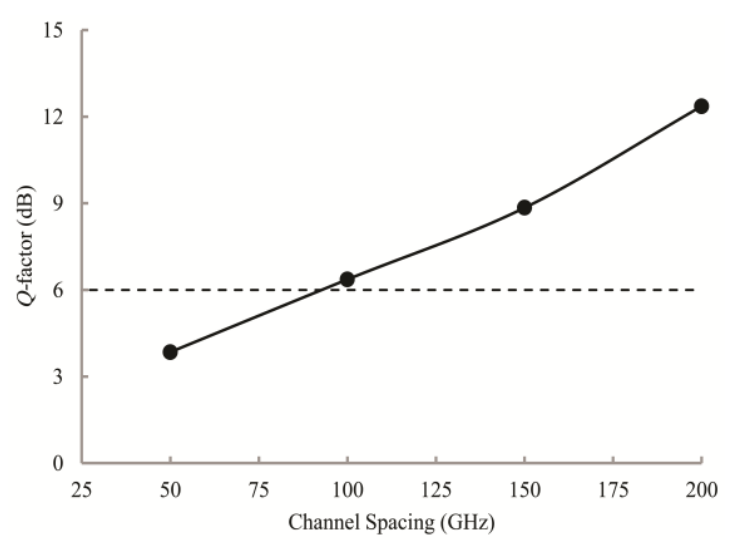

Fig. 6. $Q$-factor vs. channel spacing for 7-ch DWDM system. 
The simulation is performed for 7-ch DWDM system with transmission reach of $600 \mathrm{~km}$. The input power and bit rate per channel are taken as $3 \mathrm{~mW}$ and $40-\mathrm{Gb} / \mathrm{s}$ as usual, respectively. The system does not perform better at smaller channel spacing as inter-channel crosstalk increases. With the increase of channel spacing, the performance improves gradually. So in order to design RZ-DPSK DWDM and ultra-dense WDM (UDWDM) systems, much attention should be given to nonlinear impairments. We have to also ponder about the mitigation of nonlinear effects to maintain acceptable performance.

We have discussed the performance of RZ-DPSK DWDM system and observed the impact of XPM and FWM. Now we investigate the mitigation technique of these nonlinear effects. There are some recent works such as digital back propagation [20], inverse fiber [21] and coherent receiver [22] method on mitigation of nonlinear effects. In this paper, we use inverse fibers, i.e., negative dispersion fibers in addition to in-line DCFs. Precompensation of nonlinearity uses a model of an inverse fiber after the transmitter, so that the real transmission fiber undoes the effects of this virtual inverse fiber. Postcompensation is the same technique but it is implemented before the receiver. In pre \& post-compensation as shown in Fig. 7, inverse fibers are used in both ends. We investigate all the three compensation techniques to reduce nonlinear effects. This process is more efficient since it is done in optical domain without using any external device. Launch power is taken as $3 \mathrm{~mW}$ and transmission reach is $360 \mathrm{~km}$. We have plotted the values of $Q$ for different number of channels for uncompensated, pre-compensated, postcompensated and pre \& post- compensated transmission systems in Fig. 8. The uncompensated values were previously shown in Fig. 3. Here we find that in every case the values of $Q$ increase roughly by $1.2 \mathrm{~dB}$ for postcompensation, $2.5 \mathrm{~dB}$ for pre-compensation and $3.1 \mathrm{~dB}$ for pre $\&$ post-compensation. Thus the performance limitations due to nonlinear effects are somewhat mitigated. We also find that mitigation of nonlinear effects by pre \& post compensation is comparable to pre-compensation and it is more effective than that of post-compensation.

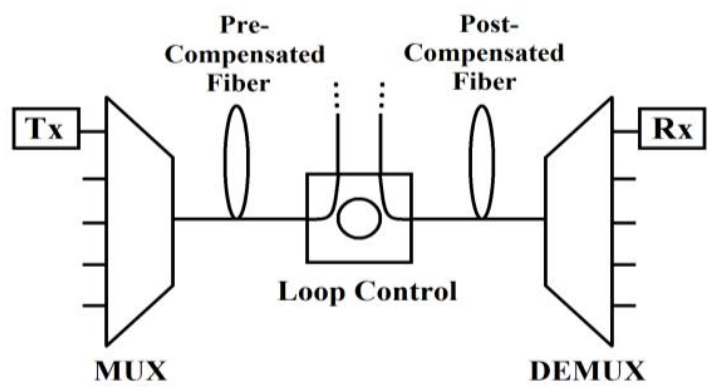

Fig. 7. An optical transmission system model with pre-compensation and/or post-compensation.

Fig. 9 shows the values of $Q$ after compensation for 24-ch at different transmission reaches for all the three compensation techniques. Since many values of $Q$ for 24-ch were less than $6 \mathrm{~dB}$, we also plot the previously shown uncompensated values for 24-ch in Fig. 4. Here for postcompensation the values increase roughly by $1.82 \mathrm{~dB}$, precompensation increases $Q$ by $2.57 \mathrm{~dB}$ and pre \& post- compensation increases $Q$ by $3.97 \mathrm{~dB}$ which support that the mitigation technique by pre $\&$ post-compensation is better than both pre-compensation and post-compensation.

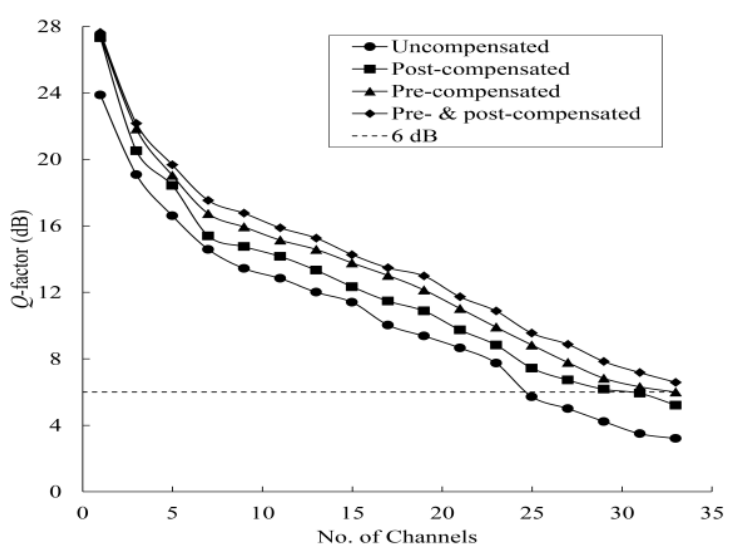

Fig. 8. $Q$-factor versus number of channels for uncompensated, postcompensated, pre-compensated and pre $\&$ post-compensated mitigation.

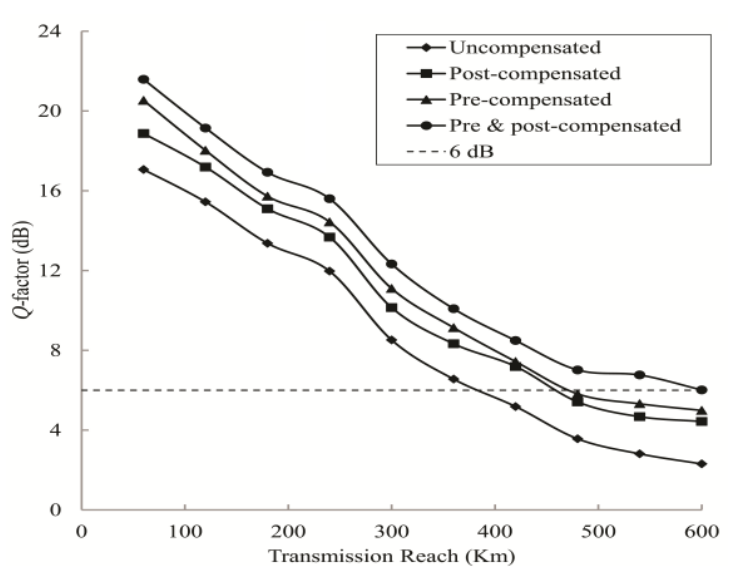

Fig. 9. $Q$-factor versus transmission reach for uncompensated, postcompensated, pre-compensated and pre \& post-compensated mitigation.

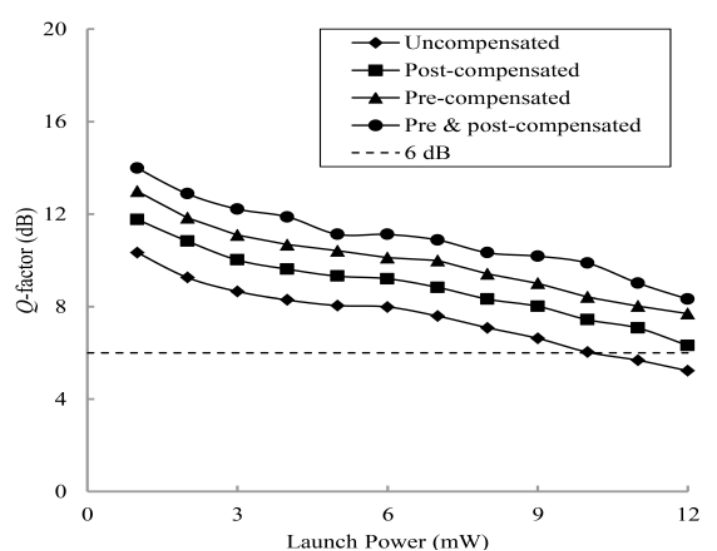

Fig. 10. $Q$-factor versus launch power for uncompensated, postcompensated, pre-compensated and pre \& post-compensated mitigation.

In the next simulation, we have considered $21-\mathrm{ch} 360 \mathrm{~km}$ long optical fiber communication system. We plot the values of $Q$ after compensation for different launch power from $1 \mathrm{~mW}$ to $12 \mathrm{~mW}$ in Fig. 10. All the three compensation techniques are investigated. Uncompensated values were previously shown in Fig. 5. Here for post-compensation the $Q$ increases by $1.45 \mathrm{~dB}$, pre-compensation increases $Q$ by $2.23 \mathrm{~dB}$ and pre \& post-compensation increases $Q$ by $3.3 \mathrm{~dB}$. In this case we also conclude that the mitigation technique by pre $\&$ post-compensation is the best. 


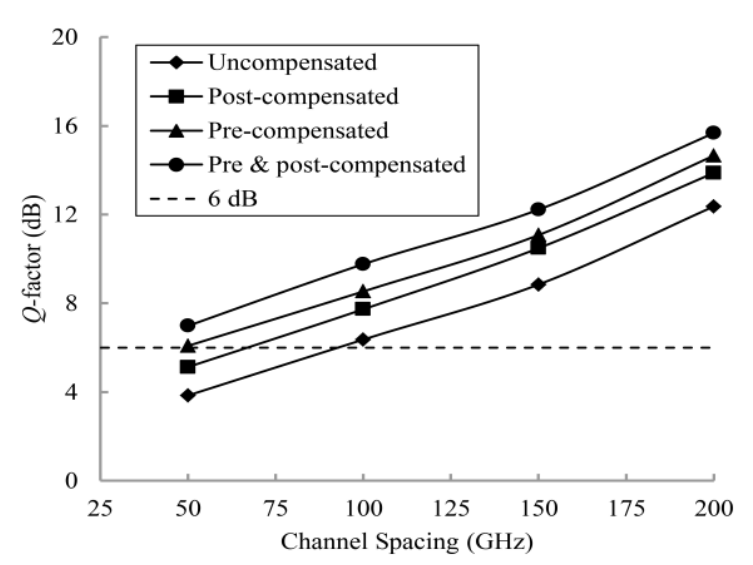

Fig. 11. $Q$-factor versus channels spacing for 7-ch uncompensated, postcompensated, pre-compensated and pre \& post-compensated DWDM system.

Our last simulation is for compensated 7-ch $600 \mathrm{~km}$ long DWDM system. All the three compensation techniques are followed. We plot uncompensated values from Fig. 6 and compensated values for channel spacing of $50 \mathrm{GHz}$ to 200 $\mathrm{GHz}$ assuming same conditions as before and the results are shown in Fig. 11. Here for post-compensation the values increase by $1.33 \mathrm{~dB}$ on average, pre-compensation increases $Q$ by $2.4 \mathrm{~dB}$ and pre $\&$ post-compensation increases $Q$ by $3.4 \mathrm{~dB}$. This investigation also shows that pre \& postcompensation technique is the best among the three.

These investigations show that all the three compensation techniques reduce fiber nonlinear effects. The reason could be the walk-off introduced by inverse fibers. The walk-off produced by compensation creates delay among the closely packed DWDM channels; as a result, XPM effect reduces. Furthermore, this compensation also introduces phase mismatch among the co-propagating channels, which in turn reduces FWM effects. However, among the three methods, pre $\&$ post-compensation is found to be the best choice.

\section{CONCLUSION}

The performance of 40-Gb/s 66\% RZ-DPSK DM DWDM systems have been investigated through numerical simulations. The transmission line has in-line amplifiers and dispersion is compensated periodically such that zero residual dispersion is preserved at the end of each span. The system performance is evaluated by varying number of channels, input power and transmission reach for both single channel and multi-channel transmission systems. Though RZ-DPSK outperforms conventional OOK and other formats, it is obvious that its performance is considerably degraded by nonlinear effects, namely XPM and FWM. To ensure optimum performance from RZ-DPSK DWDM or UDWDM, nonlinear crosstalk should be checked thoroughly while evaluating the system performance. The performance of RZ-DPSK DWDM system is also investigated employing mitigation technique to combat nonlinear effects and improved performance has been observed. Pre \& postcompensation method has been found to be much effective to mitigate nonlinear effects. Further study may include intra-channel nonlinear effects and amplifier noise etc. MUX/DEMUX filter characteristics might be investigated for higher bit rates.

\section{REFERENCES}

[1] P. J. Winzer and R. J. Essiambre, "Advanced modulation formats for high-capacity optical transport networks," J. Lightwave Technol., vol. 24, no. 12, pp. 4711-4728, 2006.

[2] F. M. Abbou, H. T. Chuah, C. C. Hiew, and A. Abid, "Comparisons of RZ-OOK and RZ-DPSK in dense OTDM-WDM systems using $Q$ factor models," J. Russian Laser Research, vol. 29, no. 2, pp. 133-141, 2008.

[3] C. Xu, X. Liu, L. F. Mollenauer, and X. Wei, "Comparison of returnto-zero differential phase-shift keying and on-off keying in long-haul dispersion managed transmission," IEEE Photon. Technol. Lett., vol. 15, no. 4, pp. 617-619, 2003.

[4] A. H. Gnauck, X. Liu, X. Wei, D. M. Gill, and E. C. Burrows, "Comparison of modulation formats for $42.7-\mathrm{Gb} / \mathrm{s}$ single channel transmission through $1980 \mathrm{~km}$ of SSMF," IEEE Photon. Technol. Lett., vol. 16, no. 3, pp. 909-911, March 2004.

[5] M. Faisal and M. H. Rahman, "Performance comparison of various PSK modulation schemes for ultra-high speed long-haul fiber-optic communication system," in Proc. IEEE Int. Conf. on Advanced Networks and Telecommunications, Bangalore, India, Dec. 16-19, 2012, pp. 96-98.

[6] M. Zaacks, U. Mahlab, P. Mamyshev, C. Rasmussen, J. Calvitti, and K. Falta, "Demonstration of $1000 \mathrm{~km} 43 \mathrm{~Gb} / \mathrm{s}$ RZ-DPSK transmission through a $50 \mathrm{GHz}$ channel spaced WSS," in Proc. OFC/NFOEC, Anaheim, CA, USA, 25-29 March, 2007, pp. 1-4.

[7] D. G. Foursa, "DPSK performance in field and laboratory experiments," in Proc. OFC/NFOEC, California, USA, 2005.

[8] C. Xu, X. Liu, and X. Wei, "Differential phase-shift keying for high spectral efficiency optical transmissions," IEEE J. Select. Topics Quantum. Electron., vol. 10, no. 2, pp. 281-293, 2004.

[9] A. H. Gnauck, G. Charlet, P. Tran, P. Winzer, C. Doerr, J. Centanni, E. Burrows, T. Kawanishi, T. Sakamoto, and K. Higuma, "25.6-Tb/s $\mathrm{C}+\mathrm{L}-\mathrm{B}$ and transmission of polarization-multiplexed RZ-DQPSK signals," in Proc. OFC/NFOEC, Anaheim, CA, 2007.

[10] J. X. Cai, C. R. Davidson, D. G. Foursa, L. Liu, Y. Cai, B. Bakhshi, G. Mohs, W. W. Patterson, P. C. Corbett, A. J. Lucero, W. Anderson, H. Li, M. Nissov, A. N. Pilipetskii, and N. S. Bergano, "Experimental comparison of the RZ-DPSK and NRZ-DPSK modulation formats," in Proc. OFC/NFOEC, vol. 4, Anaheim, CA, March 5-9, 2005,.

[11] Z. Xu, K. Rottwitt, and P. Jeppesen, "Analysis of spectral efficiency and nonlinear tolerance of DPSK formats in 160-Gb/s Raman amplified systems," IEEE Photon. Technol. Lett., vol. 17, no. 7, pp. 1552-1554, July 2005.

[12] A. Hirano and Y. Miyamoto, "Novel modulation formats in ultrahigh-speed optical transmission systems, and their applications," in Proc. OFC, Los Angeles, CA, USA, 2004.

[13] G. Lu, K. Xu, J. Wu, and J. Lin, "Performance evaluation of modulation formats in $40 \times 40-\mathrm{Gb} / \mathrm{s}$ WDM repeaterless transmission systems," Chinese Opt. Lett., vol. 4, no. 4, pp. 199-201, April 2006.

[14] W. T. Anderson, L. Liu, Y. Cai, J. X. Cai, D. Kovsh, H. Li, A. N. Pilipetskii, M. Nissov, and N. Bergano, "Modeling $40 \mathrm{~Gb} / \mathrm{s}$ CSRZDPSK and RZ-DPSK trans-Atlantic transmission with dispersion slope compensation," in Proc. OFC/NFOEC, Anaheim, CA, 5-10 March, 2006.

[15] Y. K. Lize, X. Wu, M. Nazarathy, Y. Atzmon, L. Christen, S. Nuccio, M. Faucher, N. Godbout, and A. E. Willner, "Chromatic dispersion tolerance in optimized NRZ-, RZ- and CSRZ-DPSK demodulation," Opt. Express, vol. 16, no. 6, pp. 4228-4236, March 2008.

[16] M. Daikoku, N. Yoshikane, and I. Morita, "Performance comparison of modulation formats for 40 Gbits/s DWDM transmission systems," in Proc. OFC/NFOEC, Anaheim, CA, March 6-11, 2005.

[17] A. Klekamp, R. Dischler, and W. Idler, "DWDM and single channel fibre nonlinear thresholds for $43 \mathrm{~Gb} / \mathrm{s}$ ASK and DPSK formats over various fiber types," in Proc. OFC/NFOEC, Anaheim, CA, USA, March 5-10, 2006.

[18] M. Pfennigbauer and P. J. Winzer, "Choice of MUX/DEMUX filter characteristics for NRZ, RZ, and CSRZ DWDM systems," IEEE/OSA J. Lightwave Technol., vol. 24, no. 4, 2006.

[19] G. P. Agrawal, Nonlinear Fiber Optics, 4th Ed., New York, Academic Press, 2007.

[20] T. Tanimura, S. Oda, T. Hoshida, L. Li, Z. Tao, and J. C. Rasmussen, "Experimental characterisation of nonlinearity mitigation by digital back propagation and nonlinear polarization crosstalk canceller under high PMD condition," in Proc. Conference on Optical Fiber Communication and the National Fiber Optic Engineers Conference OFC/NFOEC, Los Angeles, 2011, p. 20.

[21] A. J. Lowery, "Fiber nonlinearity mitigation in optical links that use OFDM for dispersion compensation," IEEE Photon. Technol. Lett., vol. 19 , no. 19 , pp. 1556-1558, 2007. 
[22] D. S. Millar, S. Makovejs, C. Behrens, S. Hellerbrand, R. I. Killey, P. Bayvel, and S. J. Savory, "Mitigation of fiber nonlinearity using a digital coherent receiver," J. Sel. Top. Quantum Electron., vol. 19, no. 5, pp. 1217-1226, 2010.

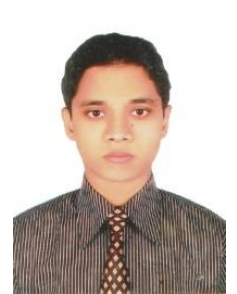

M. Arafat Rahman Khan was born in Dhaka, Bangladesh, on May 16, 1990. $\mathrm{He}$ is an undergraduate student of electrical and electronics engineering in Bangladesh University of Engineering and Technology. His major field of study is communication. His research interests include optical communication, optoelectronics and photonic networks.

At present Mr. Khan is a student member of IEEE.

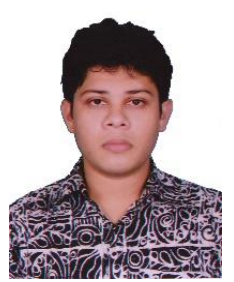

Tahsin Faruque was born in Dhaka, Bangladesh, on February 20, 1990. He is an undergraduate student of electrical and electronics engineering in Bangladesh University of Engineering and Technology. His major field of study is communication. His research interest includes optical communication and photonic networks.

Mr. Faruque is currently a student member of IEEE.

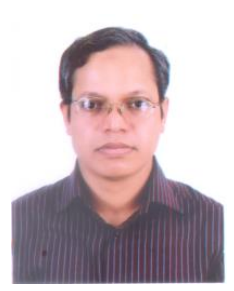

Mohammad Faisal received the B.Sc. (Hons.) and M.Sc. degrees in electrical and electronic engineering (EEE) in 2000 and 2003, respectively, from Bangladesh University of Engineering and Technology (BUET), Dhaka, Bangladesh. $\mathrm{He}$ obtained the Ph.D. degree in electrical, electronic and information engineering from Osaka University, Japan, in March 2010. His research interests include optical communication and photonic networks.

$\mathrm{He}$ has been with the Department of Electrical and Electronic Engineering, BUET, where he is currently an associate professor. He is the author and co-author of more than 24 international published papers.

Dr. Faisal is a member of the IEB and the IEEE. He is also an executive member of IEEE ComSoc Bangladesh Chapter. 